\title{
Curcumin inhibits advanced glycation end product-induced oxidative stress and inflammatory responses in endothelial cell damage via trapping methylglyoxal
}

\author{
YAN PING SUN ${ }^{1 *}$, JUN FEI GU ${ }^{2,3^{*}}$, XIAO BIN TAN ${ }^{2,3}$, CHUN FEI WANG $^{2}$, \\ XIAO BIN JIA ${ }^{2,3}$, LIANG FENG ${ }^{3}$ and JI PING LIU ${ }^{4}$
}

\begin{abstract}
${ }^{1}$ Department of Pharmacognosy, Xi'an Medical University, Xi'an, Shaanxi 710021; ${ }^{2}$ Key Laboratory of New Drug Delivery System of Chinese Materia Medica, Affiliated Hospital of Integrated Traditional Chinese and Western Medicine, Nanjing University of Chinese Medicine, Nanjing, Jiangsu 210028; ${ }^{3}$ Key Laboratory of New Drug Delivery System of Chinese Materia Medica, Jiangsu Provincial Academy of Chinese Medicine, Nanjing, Jiangsu 210028; ${ }^{4}$ Department of Pharmacology,

Shaanxi University of Chinese Medicine, Xianyang, Shaanxi 712046, P.R. China
\end{abstract}

Received January 17, 2015; Accepted September 3, 2015

DOI: $10.3892 / \mathrm{mmr} .2015 .4725$

\begin{abstract}
Methylglyoxal (MGO)-induced carbonyl stress and pro-inflammatory responses have been suggested to contribute to endothelial dysfunction. Curcumin (Cur), a polyphenolic compound from Curcuma longa L., may protect endothelial cells against carbonyl stress-induced damage by trapping dicarbonyl compounds such as MGO. However, Cur-MGO adducts have not been studied in depth to date and it remains to be known whether Cur-MGO adducts are able to attenuate endothelial damage by trapping MGO. In the present study, 1,2-diaminobenzene was reacted with MGO to ensure the reliability of the reaction system. Cur was demonstrated to trap MGO at a 1:1 ratio to form adducts 1, 2 and 3 within $720 \mathrm{~min}$. The structures of these adducts were identified by high-performance liquid chromatography/electrospray ionization tandem mass spectrometry. The kinetic curves of Cur $\left(10^{-7}, 10^{-6}\right.$ and $\left.10^{-5} \mathrm{M}\right)$ were measured from $0-168 \mathrm{~h}$ by fluorescent intensity. Cur significantly inhibited the formation of advanced glycation end products (AGEs). The differences in oxidative damage and the levels
\end{abstract}

Correspondence to: Professor Yan Ping Sun, Department of Pharmacognosy, Xi'an Medical University, 1 Xinwang Road, Xi'an, Shaanxi 710021, P.R. China

E-mail: sypgrz2000@126.com

Dr Liang Feng, Key Laboratory of New Drug Delivery System of Chinese Materia Medica, Jiangsu Provincial Academy of Chinese Medicine, 100 Shizi Street, Hongshan Road, Nanjing, Jiangsu 210028, P.R. China

E-mail:wenmoxiushi@163.com

*Contributed equally

Key words: curcumin, methylglyoxal, trapping dicarbonyl compounds, AGE generation, endothelial injury of pro-inflammatory cytokines following MGO + HSA or Cur-MGO treatment were investigated in human umbilical vein endothelial cells (HUVECs). Exposure of HUVECs to the Cur-MGO reaction adducts significantly reduced the intracellular ROS levels and improved cell viability compared with MGO alone. Furthermore, there was a significant reduction in the expression levels of transforming growth factor- $\beta 1$ and intercellular adhesion molecule-1 following treatment with Cur-MGO adducts compared with MGO alone. These results provide further evidence that the trapping of MGO by Cur inhibits the formation of AGEs. The current study indicates that the protective effect of Cur on carbonyl stress and pro-inflammatory responses in endothelial damage occurs via the trapping of MGO.

\section{Introduction}

Endothelial dysfunction has similar early pathological alterations and risk factors to those in vascular diseases, such as cardiovascular disease and diabetic vascular complications (1). Endothelium-dependent damage arises from metabolic abnormalities in the glucose metabolism that lead to vascular dysfunction (2). Carbonyl compound-induced stress and pro-inflammatory responses lead to the formation of advanced glycation end products (AGEs) (3). Methylglyoxal (MGO), a key precursor for AGEs and a reactive dicarbonyl compound, is suggested to be an intermediate derived from the metabolism of glucose (4). Numerous previous studies support a role for MGO in triggering two distinct signaling cascades leading to oxidative damage and pro-inflammatory responses in HUVECs $(5,6)$. The trapping of dicarbonyl compounds like MGO represents an effective strategy for attenuating carbonyl stress-induced endothelial injury (3).

Curcumin (Cur) is the most active component of the curcuminoids extracted from Curcuma longa L. and has been demonstrated to protect against AGE-induced cellular inflammatory responses and oxidative stress in vascular complications $(7,8)$. Increasing evidence has suggested that Cur 
possesses a potential protective effect against MGO-induced endothelial dysfunction via scavenging reactive oxygen species (ROS) and attenuating the levels of inflammatory mediators (9). Previous studies have indicated that Cur may prevent MGO-induced endothelial dysfunction by directly trapping MGO to form a curcumin-MGO adduct at the electron-dense carbon atom $(\mathrm{C} 10)$ between the two keto carbon groups (10). However, Cur-MGO adducts have not been studied in depth at present and it remains to be investigated whether Cur-MGO adducts are able to attenuate cytotoxicity in HUVECs.

In the present study, the trapping capacity of Cur was investigated through examining the optimal reaction parameters and analyzing the Cur-MGO adducts by high-performance liquid chromatography-diode-array detection (HPLC-DAD) coupled with liquid chromatography-electrospray ionization-tandem mass spectrometry (LC-ESI-MS/MS). Furthermore, the differences in the levels of oxidative damage and pro-inflammatory cytokines as a result of the formation of AGEs in the presence or absence of Cur were compared in HUVECs to investigate the protective mechanisms of Cur on endothelial dysfunction.

\section{Materials and methods}

Chemicals and materials. MGO, 40\% aqueous solution), aminoguanidine hydrochloride (AG; purity $\geq 98 \%$ ), 3-(4,5-dimethylthiazol-2-yl)-2,5-diphenyltetrazolium bromide (MTT), 1,2-diaminobenzene (DB) and 2-methylquinoxaline were purchased from Sigma-Aldrich (St. Louis, MO, USA). Human serum albumin (HSA) was obtained from Amresco LLC (Solon, OH, USA). Human AGEs ELISA kit, SABC kit, transforming growth factor- $\beta 1$ (TGF- $\beta 1$; cat. no. sc-1672; 1:200) and intercellular adhesion molecule-1 (ICAM-1; cat. no. sc-1506; 1:200) antibodies were obtained from Santa Cruz Biotechnology, Inc., (Dallas, TX, USA). The ROS kit was purchased from Beyotime Institute of Biotechnology (Nantong, China). Curcumin (purity $\geq 98 \%$ ) was obtained from the National Institute for the Control of Pharmaceutical and Biological Products (Beijing, China). HPLC-grade methanol was purchased from Tedia (Fairfield, OH, USA). HPLC-grade water was prepared using a Millipore Milli-Q purification system (EMD Millipore, Billerica, MA, USA). Other reagents were analytical grade and from Nanjing Chemical Reagent Co., Ltd. (Nanjing, China).

Cell culture. Human umbilical vein endothelial cells (HUVECs), were selected to model endothelial disease in vitro and were purchased from the American Type Culture Collection (Manassas, VA, USA). HUVECs were cultured in low-glucose Dulbecco's modified Eagle's medium supplemented with $10 \%$ fetal bovine serum (FBS; Gibco Life Technologies, Carlsbad, CA, USA), $80 \mathrm{U} / \mathrm{ml}$ penicillin and $80 \mathrm{U} / \mathrm{ml}$ streptomycin. The cells were maintained in a humidified incubator at $37^{\circ} \mathrm{C}$ containing $5 \% \mathrm{CO}_{2}$. The culture medium was replaced every 2 days. HUVECs used in the study were passaged 3-5 times prior to use.

Reaction of Cur or DB with MGO. DB (1 mM) and MGO (1 mM) were incubated in $2 \mathrm{ml}$ phosphate-buffered saline (PBS; 0.2 M, pH 7.4; Nanjing KeyGen Biotech Co., Ltd., Nanjing, China). The mixtures were agitated at $40 \mathrm{rpm}$ at $37^{\circ} \mathrm{C}$ for 30, 90, 240 and $720 \mathrm{~min}$. A PBS solution was used as the blank control. Cur $(1 \mathrm{mM})$ and MGO $(1 \mathrm{mM})$ were incubated in $0.2 \mathrm{M}$ PBS. The mixtures were agitated at $40 \mathrm{rpm}$ at $37^{\circ} \mathrm{C}$ for $720 \mathrm{~min}$. MGO alone $(1 \mathrm{mM})$ or Cur $(1 \mathrm{mM})$ was used as the control. Following the reaction, the samples were dried using high purity nitrogen overnight. The residue was redissolved in $1 \mathrm{ml} \mathrm{HPLC}$-grade methanol and vortexed for $30 \mathrm{sec}$. The sample solutions were filtered through a $0.45 \mu \mathrm{m}$ microporous membrane prior to HPLC analysis.

Kinetic study of the trapping of $M G O$ by Cur. In order to optimize the incubation ratio of Cur and MGO, Cur $(0.2,0.33$, 1, 3 and $5 \mathrm{mM}$ ) was incubated with $1 \mathrm{mM}$ MGO in $3 \mathrm{ml}$ PBS $(0.2 \mathrm{M}, \mathrm{pH} 7.4)$ at $37^{\circ} \mathrm{C}$ and agitated at $40 \mathrm{rpm}$. Subsequently, $200 \mu \mathrm{l}$ reaction mixture was collected at each time point, $1 \mu \mathrm{l}$ acetic acid (Nanjing Chemical Reagent Co., Ltd.) added to stop the reaction and $100 \mathrm{mM}$ DB $(14.6 \mathrm{mmol})$ added to react with the remaining $\mathrm{MGO}$, as previously described (11).

HPLC analysis of the reaction mixture of Cur or DB with MGO. HPLC-DAD was conducted to analyze the reaction mixture of Cur or DB with MGO. This analysis was conducted on an Agilent 1200 system (Agilent Technologies, Inc., Santa Clara, CA, USA) which was equipped with a quaternary pump, DAD detector, an autosampler and Agilent ChemStation B.0401 software. The analysis conditions were as follows: An Alltima C18 $(4.6 \times 150 \mathrm{~mm}, 5 \mu \mathrm{m})$ column was used for this analysis; the flow rate was set at $1.0 \mathrm{ml} / \mathrm{min}$ and the column temperature maintained at $30^{\circ} \mathrm{C}$. For the reaction of Cur and $\mathrm{MGO}$, the mobile phase consisted of methanol (eluent A) and water (eluent B) with a gradient program of $0-45 \mathrm{~min}, 2-80 \% \mathrm{~A}$, conducted for component separation with a linear gradient elution, and the determination wavelength set at $425 \mathrm{~nm}$. For the reaction of $\mathrm{DB}$ and $\mathrm{MGO}$, methanol (eluent $\mathrm{A}$ ) and water (eluent B), (0-9 min, 5-50\% A) was used for the mobile phase with a wavelength of $280 \mathrm{~nm}$ set. In total, a $20 \mu 1$ sample was injected into the HPLC system.

LC-ESI-MS/MS analysis. An Agilent 1200 HPLC system combined with an LCQ-Fleet Ion Trap Mass Spectrometer (Thermo Fisher Scientific, Waltham, MA, USA) was used to identify the chemical structure of the Cur-MGO adducts. The detailed conditions and parameters were as follows: The ionization was achieved using the electrospray in positive mode; helium was used as the collision gas and nitrogen $\left(\mathrm{N}_{2}\right)$ as the nebulizing gas; spray voltage was set at $4.5 \mathrm{kV}$ and capillary voltage at $5 \mathrm{~V}$; the capillary temperature was kept at $300^{\circ} \mathrm{C} ; \mathrm{N}_{2}$ was selected as the sheath gas and its pressure was set at 90 arbitrary units. In addition, the isolation width of the precursor ions was set at $1.5 \mathrm{Th}$. The mass range of compounds scanned was from $50-1,200 \mathrm{~m} / \mathrm{z}$.

$M G O$ derived-AGEs preparation in the presence or absence of Cur. MGO-AGEs were prepared with a modified reaction quantity of HSA and MGO as previously described (12). Briefly, $4 \mathrm{mg}$ HSA and $50 \mathrm{mM}$ MGO were incubated in $1 \mathrm{ml}$ PBS (0.2 M, pH 7.4) in the presence or absence of Cur $\left(10^{-7}, 10^{-6}\right.$ and $\left.10^{-5} \mathrm{M}\right)$ under sterile conditions and maintained in $95 \%$ air $/ 5 \% \mathrm{CO}_{2}$ at $37^{\circ} \mathrm{C}$. For the kinetic study, the mixture was agitated for $0,4,12,24,48,72,96,120,144$ and $168 \mathrm{~h}$. 
Similarly, HSA in the absence of MGO was incubated in the same conditions. AG $\left(10^{-6} \mathrm{M}\right)$ was selected as the positive control. The fluorescent intensity of MGO-AGEs was determined using a fluorescent microplate reader with the excitation/emission wavelength set at 370/440 nm (Gemini EM $^{\mathrm{TM}}$; Molecular Devices, LLC, Sunnyvale, CA, USA) (13). In addition, the level of MGO-AGEs was measured using an AGEs ELISA kit and a SpectraMax 190 microplate reader at $450 \mathrm{~nm}$ (Molecular Devices, LLC). The obtained MGO-AGEs were stored at $4^{\circ} \mathrm{C}$ for further experimental use. MGO-AGEs containing $1 \mathrm{mM}$ MGO were used for the cell experiments.

ELISA assay for MGO-AGEs. In order to measure the inhibitory effect of Cur on the formation of MGO-AGEs, an Human AGE ELISA kit (cat. no. sc-1609; Santa Cruz Biotechnology, Inc.) was used to measure the level of MGO-AGEs, according to the manufacturer's instructions. The optical density (OD) value of samples was measured using an MK-3 microplate reader (Thermo Fisher Scientific, Inc.) at $450 \mathrm{~nm}$.

Dihydroethidium (DHE) staining and flow cytometry for the level of ROS. MGO-AGEs may induce intracellular ROS generation and lead to endothelial injury. Therefore, in the current study, DHE (Nanjing KeyGen Biotech Co., Ltd.) staining was conducted to measure the level of ROS as previously described (14). Cells were incubated with $5 \mu \mathrm{M}$ DHE at $37^{\circ} \mathrm{C}$ for $30 \mathrm{~min}$ according to the manufacturer's instructions. The free DHE molecules were then removed by washing with PBS. Fluorescent images of ROS were captured by fluorescence microscopy (Olympus IX73; Olympus Corporation, Tokyo, Japan). In addition, the level of intracellular ROS was measured using flow cytometery (FACSCalibur; BD Biosciences, Franklin Lakes, NJ, USA) at an excitation/emission wavelength of $488 / 530 \mathrm{~nm}$ (15). The fluorescence intensity of DHE was analyzed in 10,000 cells using CellQuest software (BD Biosciences).

MTT assay for cell viability. To investigate the cytotoxicity of the Cur-MGO adducts, an MTT assay was used to measure the cell viability of HUVECs. Cells $\left(1 \times 10^{5}\right.$ cells/well) were seeded into 96 -well plates and incubated for $24 \mathrm{~h}$. Once $80 \%$ confluent, cells were stimulated with Cur-MGO adducts for $48 \mathrm{~h}$. Subsequently MTT ( $5 \mathrm{mg} / \mathrm{ml}, 10 \mu \mathrm{l})$ was added in FBS-free media to each well to replace the original media and then incubated for $4 \mathrm{~h}$ at $37^{\circ} \mathrm{C}$ in an incubator. Following this, the supernatant was removed and the formazan crystals were dissolved in $100 \mu 1$ dimethyl sulfoxide (Nanjing Chemical Reagent Co., Ltd.). Following agitation for $10 \mathrm{~min}$, the OD of each sample was measured using the microplate reader at $550 \mathrm{~nm}$. Cell viability was calculated according to the relative difference in OD value.

Immunocytochemistry for ICAM-1 and TGF- $\beta 1$. Immunocytochemistry was conducted to investigate the effect of Cur-MGO adducts on the expression levels of ICAM-1 and TGF- $\beta 1$ in HUVECs (16). Cells were grown on glass coverslips in 24-well plates. Following $48 \mathrm{~h}$ stimulation with Cur-MGO adducts, HUVECs were fixed in fresh $4 \%$ formaldehyde (Nanjing Chemical Reagent Co., Ltd.) and washed three times in PBS. Cells were treated with $3 \% \mathrm{H}_{2} \mathrm{O}_{2}$ for
10 min to block endogenous peroxidase activity. Subsequently, cells were blocked with $5 \%$ bovine serum albumin (BSA; Roche Diagnostics, Basel, Switzerland) for $30 \mathrm{~min}$ and then incubated with ICAM-1 (1:500) or TGF- $\beta 1$ (1:500) antibodies for $120 \mathrm{~min}$ at $37^{\circ} \mathrm{C}$. Cells were then incubated with biotin-conjugated secondary antibodies ICAM1 and TGF- $\beta 1$ for $120 \mathrm{~min}$ at room temperature, and the staining was visualized using 3,3-diaminobenzidine (Nanjing KeyGen Biotech Co., Ltd.). The cells were counterstained with hematoxylin (Nanjing KeyGen Biotech Co., Ltd.) and images were captured using a microscope (Olympus IX71; Olympus Corporation) and Image-Pro Plus 6.0 software (Media Cybernetics, Inc., Rockville, MD, USA). As a control, the primary antibodies were replaced with PBS.

Western blot analysis for ICAM-1 and TGF- $\beta 1$ levels. Following treatment with Cur-MGO adducts, protein was extracted from HUVECs using lysis buffer containing 1\% Triton X-100 (Nanjing Chemical Reagent Co., Ltd.), $150 \mathrm{mM} \mathrm{NaCl}$ (Nanjing Chemical Reagent Co., Ltd.), $1 \mathrm{mM}$ EDTA (Nanjing KeyGen Biotech Co., Ltd.), 20 mM Tris-HCl (Nanjing KeyGen Biotech Co., Ltd.), $5 \mu \mathrm{g} / \mathrm{ml}$ pepstatin A (Nanjing KeyGen Biotech Co., Ltd.), 2 mM diisopropyl fluorophosphate (Nanjing Chemical Reagent Co., Ltd.) and $1 \mathrm{mM}$ phenylmethylsulfonyl fluoride (Nanjing Chemical Reagent Co., Ltd.). Equal amounts of protein $(50 \mu \mathrm{g})$ were loaded and then separated using 10\% SDS-PAGE (Nanjing KeyGen Biotech Co., Ltd.). Subsequently, the proteins were transferred to polyvinylidene difluoride membranes (EMD Millipore). Following blocking with 5\% BSA in Tris-buffered saline containing 0.1\% Tween-20 (Nanjing KeyGen Biotech Co., Ltd.) for $1 \mathrm{~h}$, the membranes were incubated with primary antibodies against ICAM-1 (1:500) or TGF- $\beta 1(1: 500)$ at $37^{\circ} \mathrm{C}$ for $2 \mathrm{~h}$. Following three washes with PBS, the membranes were incubated with horseradish peroxide-conjugated ICAM1 and TGF- $\beta 1$ secondary antibodies $(1: 1,000)$ for $2 \mathrm{~h}$ at $37^{\circ} \mathrm{C}$. Bands were visualized using enhanced chemiluminescence (Nanjing KeyGen Biotech Co., Ltd.) and imaged using a minicamera (Olympus IX71). The experiment was repeated a minimum of three times.

Statistical analysis. All data were taken from three individual experiments and presented as the mean \pm standard deviation. SPSS software, version 16.0 (SPSS, Inc., Chicago, IL, USA) was used to conduct statistical analyses using a one-way analysis of variance and Tukey's test. $\mathrm{P}<0.05$ was considered to indicate a statistical significance difference.

\section{Results}

The reliability of the reaction system. To ensure the reliability of the reaction system, DB was reacted with MGO. The aldehyde groups of $\mathrm{MGO}$ and the amino groups of $\mathrm{DB}$ form $-\mathrm{CH}=\mathrm{N}$ - through a Schiff base reaction, forming 2-methylquinoxaline (Fig. 1A). Following the reaction, the 2-methylquinoxaline generated by the reaction system was analyzed by HPLC-DAD at $280 \mathrm{~nm}$. As presented in Fig. 1B, the reaction product matched the 2 -methylquinoxaline standard according to the UV spectra and retention times. In order to investigate the optimal reaction time, 30, 90, 
A<smiles>CC(=O)C=O</smiles>

MGO<smiles>Nc1ccccc1N</smiles>

DB<smiles>Cc1cnc2ccccc2n1</smiles>

2-methylquinoxaline
$\mathbf{B}$

a

b
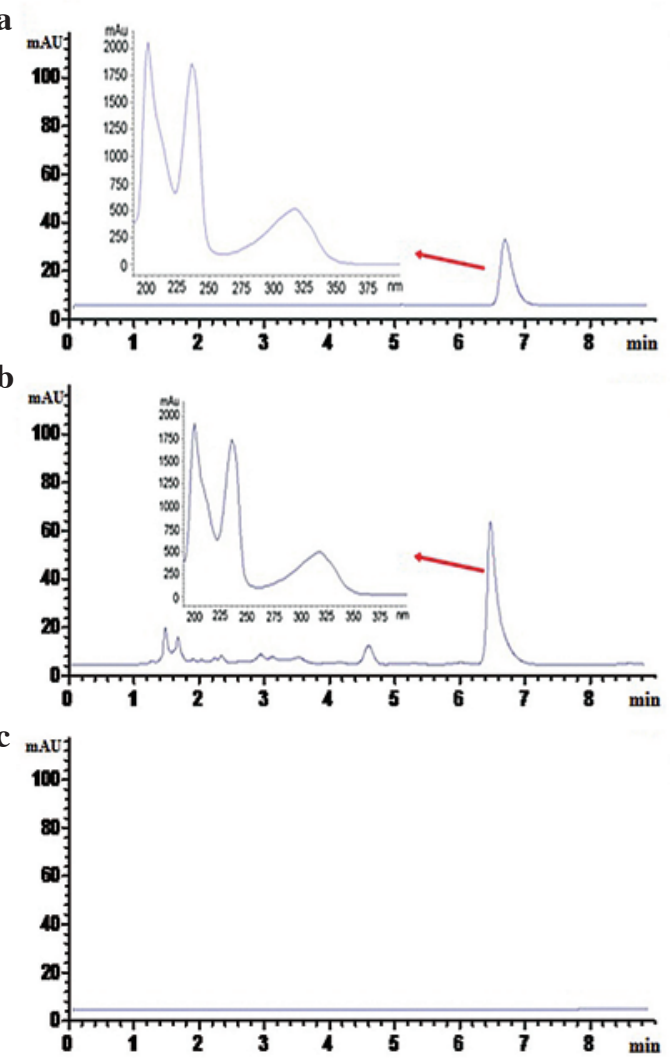

C
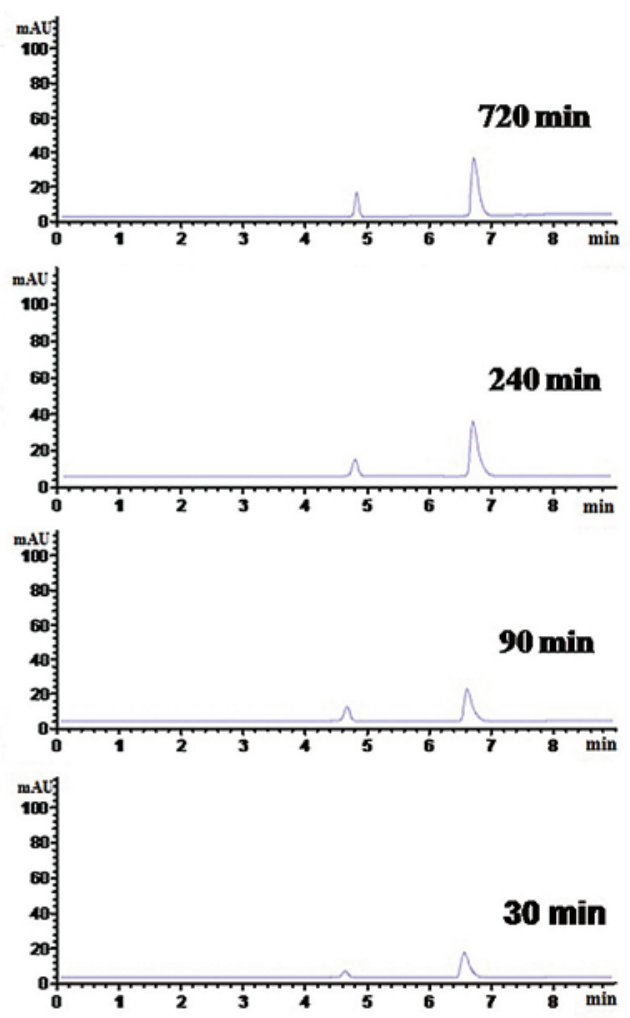

Figure 1. Reliability of the reaction system of MGO and Cur. DB (1 mM) was reacted with MGO (1 mM) in PBS solution to form 2-methylquinoxaline. (A) The chemical reaction of MGO and DB. (B) High-performance liquid chromatography analysis (280 $\mathrm{nm}$ wavelength) of 2-methylquinoxaline derived from MGO and DB (a, the reaction product; b, 2-methylquinoxaline standard; c, PBS). (C) The formation of 2-methylquinoxaline in the reaction system at different time points. MGO, methylgloxal; Cur, curcumin; DB, 1,2-diaminobenzene; PBS, phosphate-buffered saline.
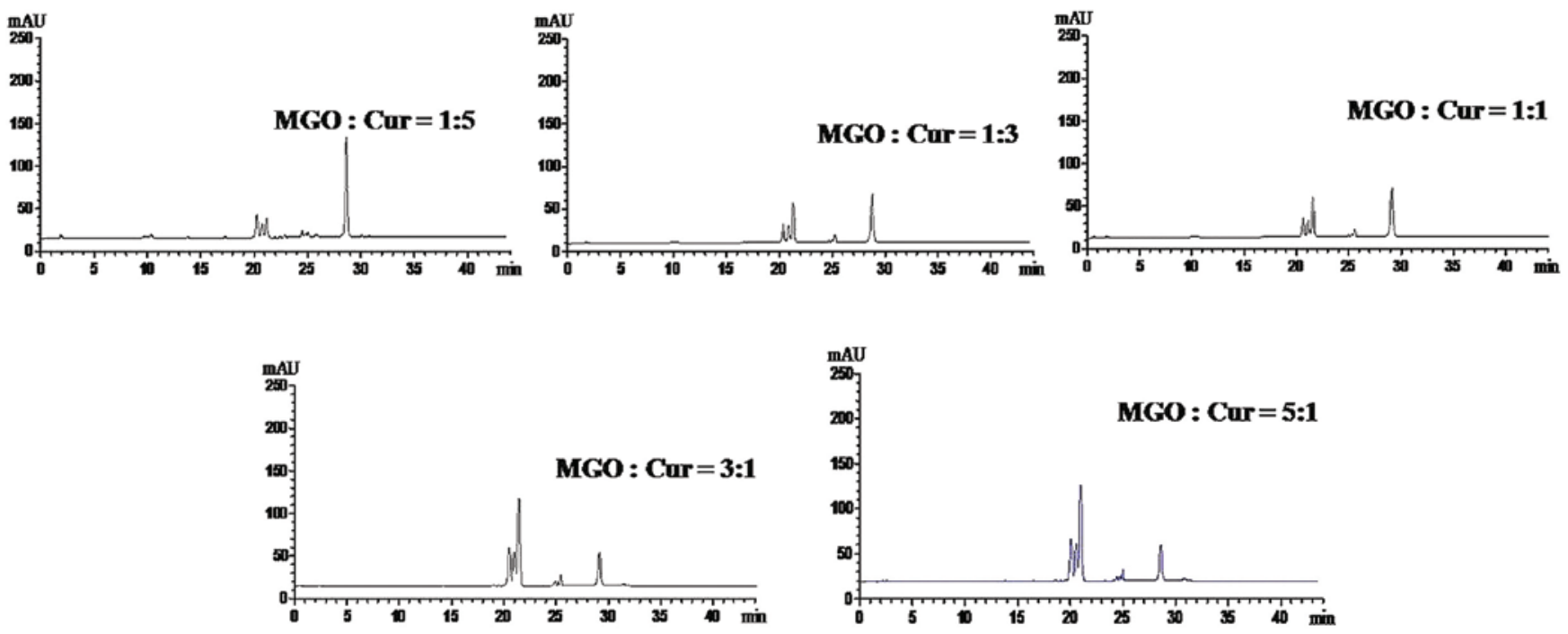

Figure 2. Screening for the optimal reaction ratio of $\mathrm{Cur}$ and MGO. The reaction was conducted in the phosphate-buffered saline solution system $\left(\mathrm{pH} 7.4,37^{\circ} \mathrm{C}\right.$ ) containing MGO:Cur at ratios of 1:5, 1:3, 1:1, 3:1 and 5:1 for 720 min. Cur, curcumin; MGO, methylgloxal. 
A

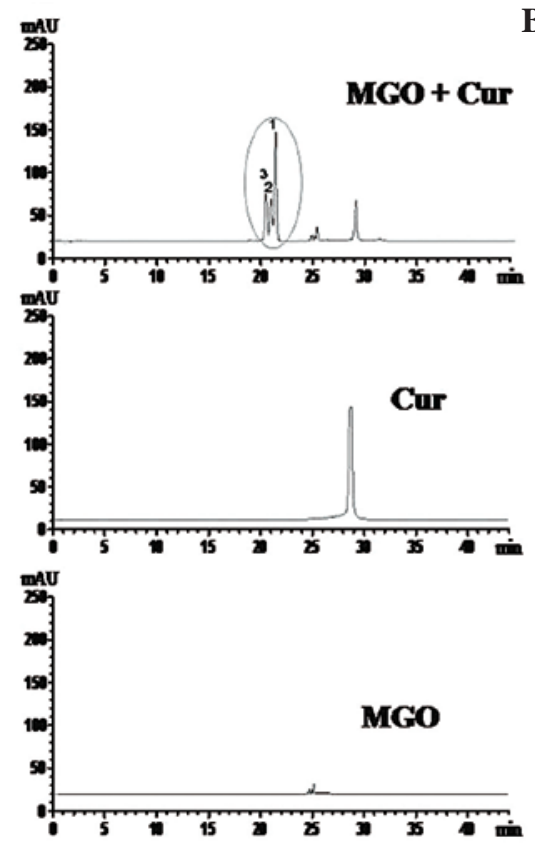

B

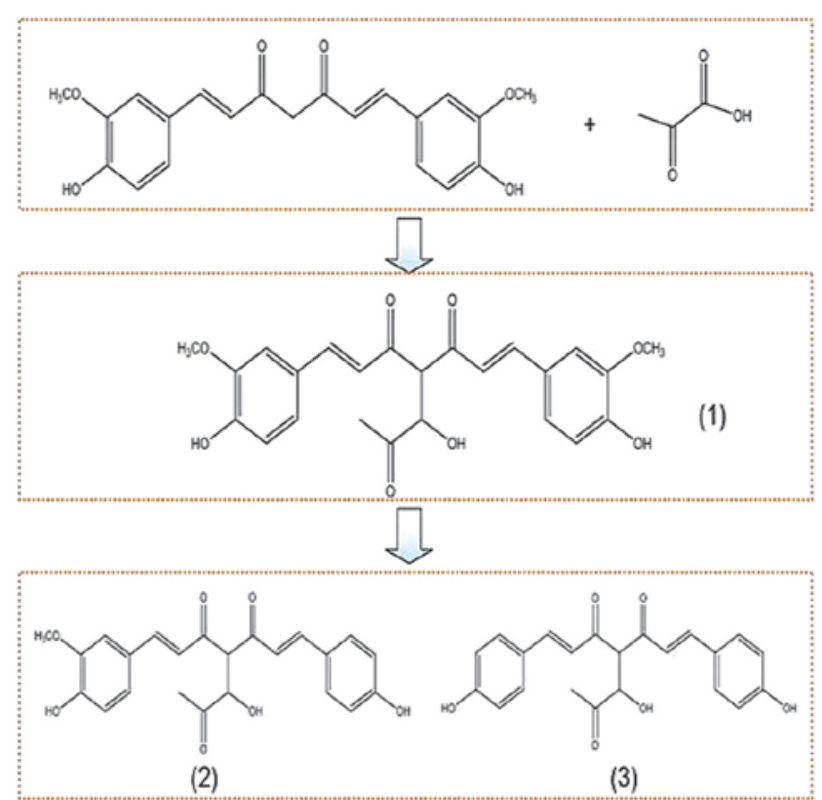

Figure 3. Trapping ability of Cur on MGO in (A) the reaction system and (B) the proposed formation pathway. MGO (1 mM) was reacted with Cur $(1 \mathrm{mM})$ in phosphate-buffered saline solution for $720 \mathrm{~min}$. The compounds formed from the reaction system were detected by high-performance liquid chromatography/diode-array detection at $425 \mathrm{~nm}$. MGO or Cur alone were selected to be compared with the generated compounds. Cur, curcumin; MGO, methylgloxal.

240 and 720 min were selected as reaction time points. The HPLC-DAD indicated that the level of 2-methylquinoxaline in the reaction system increased gradually and that the content reached the maximum level at $720 \mathrm{~min}$ (content at later time points was measured in preliminary experiments). Therefore, 720 min was selected as the optimal reaction time. The results indicated that the reaction system was suitable for trapping dicarbonyl compounds.

The optimal reaction ratio for the trapping of $M G O$ by Cur. In order to identify the optimal reaction conditions, the ratio of MGO:Cur (1:5, 1:3, 1:1, 3:1 and 5:1) was investigated. As presented in Fig. 2, the content of Cur in the reaction system was reduced and three novel peaks were increased with the increasing MGO:Cur ratio. When the ratio of MGO:Cur was 1:1, the content of Cur was not further reduced while the formation of MGO-Cur adducts was not increased. This indicated that the 1:1 ratio of MGO:Cur resulted in a complete chemical reaction.

Identification of Cur-MGO adducts by HPLClelectrospray ionization tandem mass spectrometry (HPLC-ESI-MS/MS). The ability of Cur to capture the carbonyl compound MGO was investigated in the reaction system. MGO $(1 \mathrm{mM})$ and Cur $(1 \mathrm{mM})$ were reacted together for $720 \mathrm{~min}$. As presented in Fig. 3A, compared with Cur alone or MGO alone, three new peaks were formed on the chromatogram along with a reduction in the Cur peak. The retention time of Cur was 29.16 min while the three Cur-MGO adducts were 20.17, 20.94 and $21.37 \mathrm{~min}$. These results demonstrated that Cur is able to trap MGO to form three Cur-MGO adducts.

To identify the Cur-MGO adducts, HPLC-ESI-MS/MS was conducted to analyze the molecular ion composition. As presented in Fig. 4, Cur-MGO adducts exhibited similar retention times of pure Cur and the molecular ion mass to charge ratios $(\mathrm{m} / \mathrm{z})$ of 339, 309, 293 and 217 [(M+H)-]. Peak 1 was identified as Cur-MGO adduct 1 according to the fragment ion $\mathrm{m} / \mathrm{z}$ of $411,381,364,289$ and 217 [(M+H)-]. Adduct 1 is the same as a previously reported adduct, with a $\beta$-hydrogen shift to the double bond of one of the diketones of deprotonated Cur (10). In addition, the current study identified two novel Cur-MGO adducts, adducts 2 and 3 . Based on the fragment ion $\mathrm{m} / \mathrm{z}$ of $411,381,365,349,273$ and 217, and 381, 365, 349, 273 and 201, adducts 2 and 3 were suggested to be present due to the observation of the loss of one or two $-\mathrm{OCH}_{3}$ groups, respectively. The formation of these adducts may be associated with the structural instability of Cur and Cur-MGO adducts. The potential formation pathway under physiological conditions of Cur-MGO adducts is presented in Fig. 3B ( $\mathrm{pH} \mathrm{7.4,}$ $\left.37^{\circ} \mathrm{C}\right)$.

Inhibitory effect of Cur on the formation of AGEs. In order to further investigate the inhibitory effect of Cur on the formation of AGEs through the trapping of dicarbonyl compounds, Cur was incubated with MGO and HSA and the reaction kinetics were measured. As presented in Fig. 5A, there was a reduction in the formation of MGO-AGEs with increasing concentrations of Cur $\left(10^{-7}-10^{-5} \mathrm{M}\right)$. Following incubation for $24 \mathrm{~h}$, the relative fluorescence units of the samples in the presence or absence of Cur were stable. Furthermore, AG, the positive control $\left(10^{-6} \mathrm{M}\right)$, demonstrated a significant inhibition of the formation of MGO-AGEs. In addition, the results of the ELISA demonstrated that Cur was able to significantly inhibit the formation of MGO-AGEs in a concentration-dependent manner, compared with the MGO and MGO + HSA groups $(\mathrm{P}<0.01$; Fig. 5B and $\mathrm{C})$. 
A

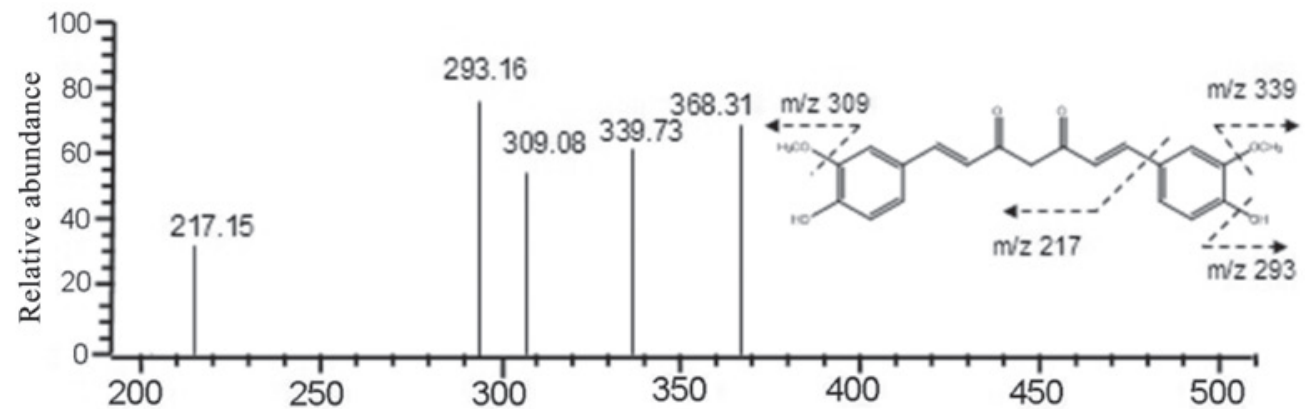

B

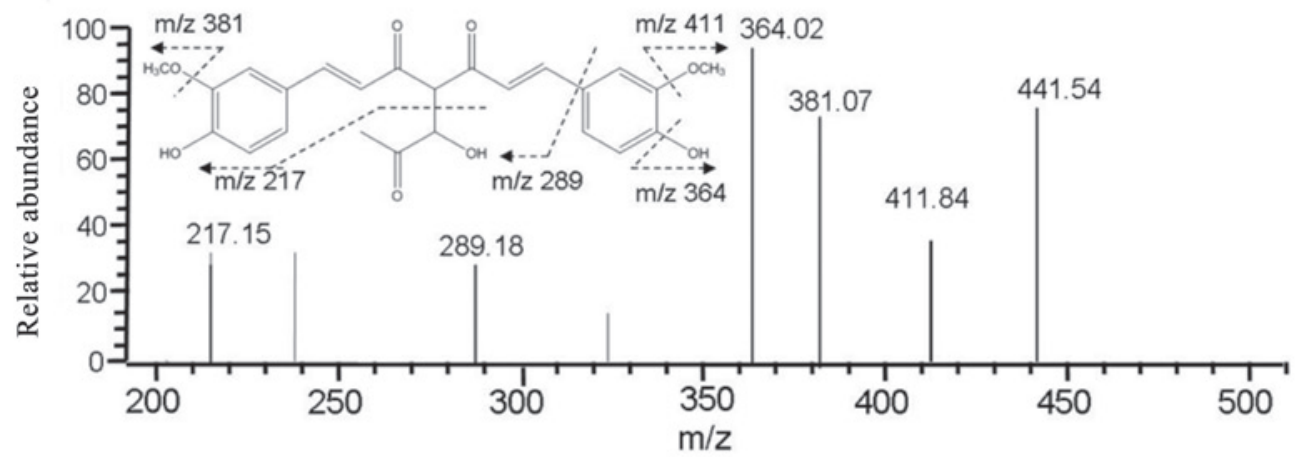

C

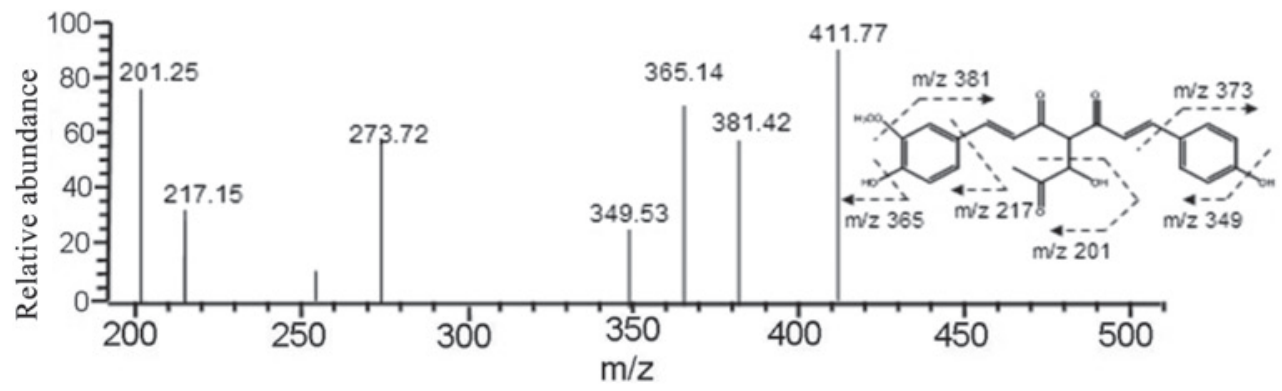

D

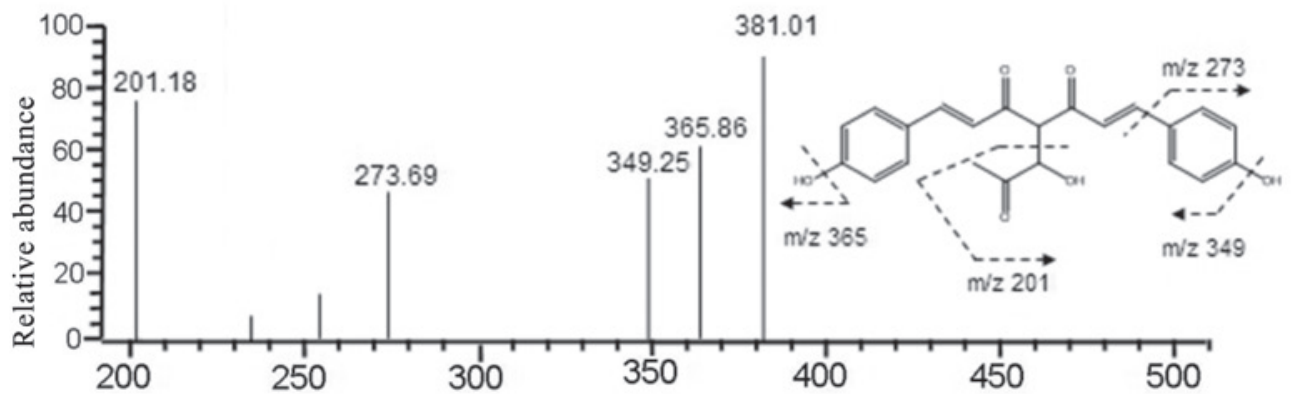

Figure 4. Identification of curcumin-methylglyoxal adducts using high-performance liquid chromatography/electrospray ionization tandem mass spectrometry. (A) Curcumin; (B) adduct 1; (C) adduct 2; (D) adduct 3.

These results indicate that Cur inhibits the formation of MGO-AGEs in the reaction system.

Attenuation of ROS generation by Cur-MGO adducts in HUVECs. The level of ROS in HUVECs was measured using a ROS kit and flow cytometry. As presented in Fig. 6A, the production of ROS was significantly induced by MGO or MGO + HSA $(\mathrm{P}<0.01)$. However, the generation of ROS was inhibited following incubation with Cur-MGO adducts in a concentration-dependent manner. Quantification of the flow cytometry results demonstrated that the Cur-MGO adducts resulted in a significant reduction in the generation of ROS $(\mathrm{P}<0.05$ or $\mathrm{P}<0.01)$ (Fig. $6 \mathrm{~B}$ and $\mathrm{C}$ ). These data demonstrate that Cur may attenuate MGO-induced endothelial dysfunction via the trapping of MGO.

Reduced cytotoxicity of HUVECs treated with Cur-MGO adducts. An MTT assay was conducted to measure the cell viability of HUVECs in order to investigate the role of Cur in the protection against MGO-induced cell injury. Following exposure to MGO or MGO + HSA, the cell viability of HUVECs was reduced compared with HSA treatment only $(\mathrm{P}<0.05)$. Notably, this reduction was restored to normal levels by Cur-MGOs $\left(10^{-7}, 10^{-6}\right.$ and $\left.10^{-5} \mathrm{M}\right)$. In addition, the Cur-MGO adducts resulted in reduced cytotoxicity compared with MGO + HSA. Furthermore, the effect of Cur was similar 


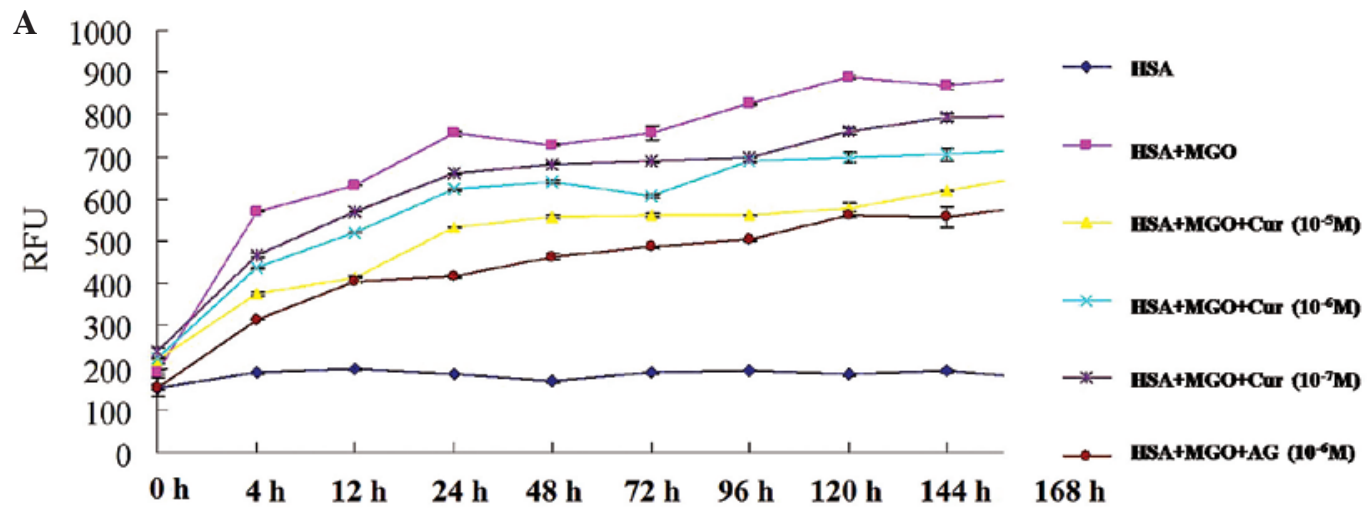

B
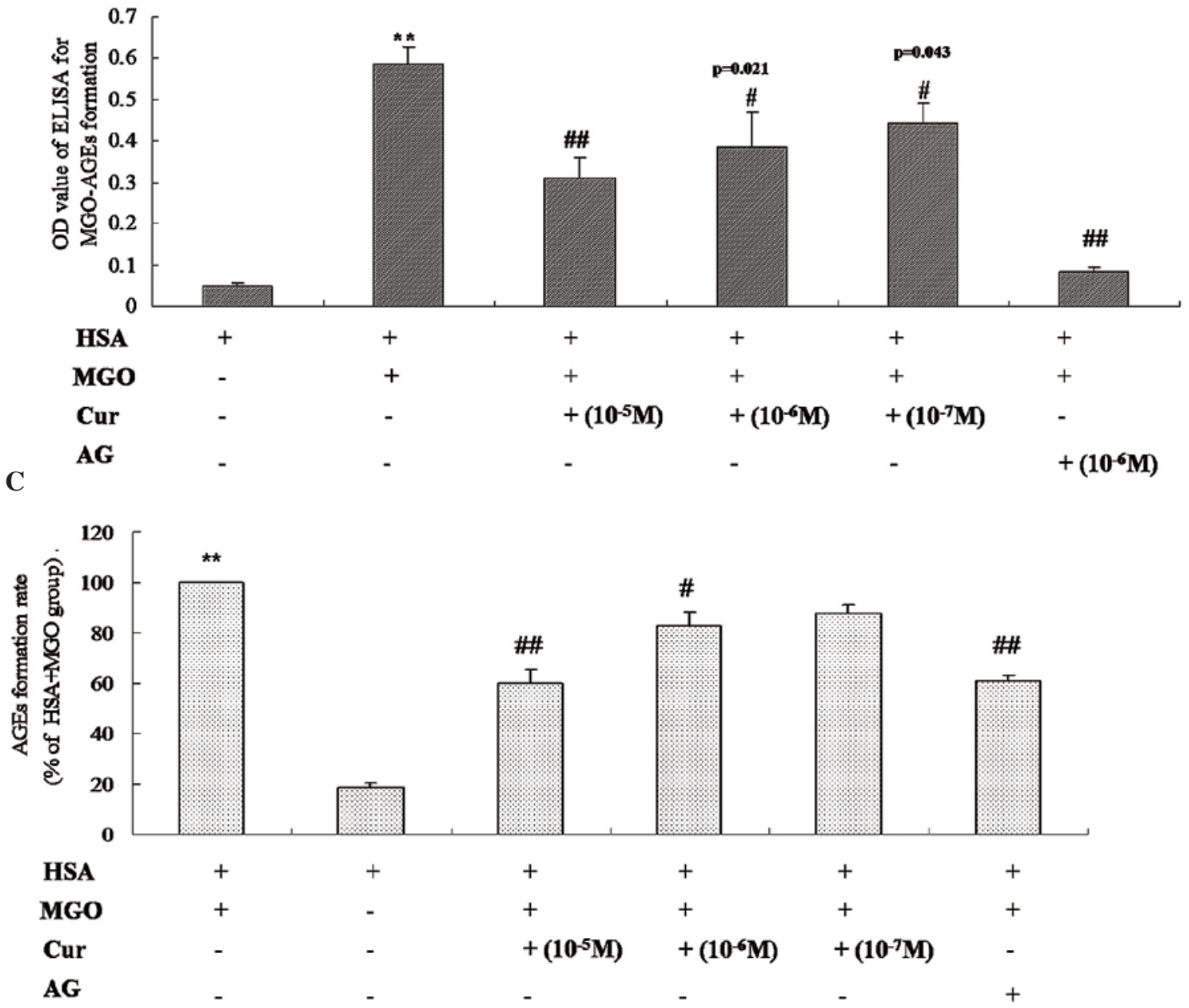

Figure 5. Inhibitory effect of Cur on the formation of AGEs in the MGO and HSA reaction system. Cur $\left(10^{-7}, 10^{-6}\right.$ and $\left.10^{-5} \mathrm{M}\right)$ was co-incubated with MGO $(50 \mathrm{mM})$ and HSA $(4 \mathrm{mg})$ in $1 \mathrm{ml}$ phosphate-buffered saline solution for 0,4,12, 24, 48, 74, 96, 120, 144 and $168 \mathrm{~h}$. (A) The fluorescent intensity of MGO-AGEs was determined at the excitation/emission wavelengths of 370/440 nm. (B) The level of MGO-AGEs was measured using ELISA and (C) the relative formation rate of MGO-AGEs was calculated. The formation rate of MGO-AGEs was calculated according to the following formula: The formation rate $=[(\mathrm{OD}$ MGO \& HSA - OD MGO \& HSA \& Cur)/OD MGO \& HSA] x 100 . AG $\left(10^{-6} \mathrm{M}\right)$ was used as the positive control. The data are presented as the mean \pm standard deviation $(\mathrm{n}=6) .{ }^{* *} \mathrm{P}<0.01$, vs. HSA or vs. MGO + MGO; ${ }^{\#} \mathrm{P}<0.01,{ }^{\# \#} \mathrm{P}<0.01$, vs. MGO + HSA. Cur, curcumin; AGEs, advanced glycation end products; MGO, methylgloxal; HSA, human serum albumin; OD, optical density; AG, aminoguanidine hydrochloride; RFU, relative fluorescence units.

to that of the AG postive control $\left(10^{-6} \mathrm{M}\right.$; Fig. 7). These data suggest that Cur may attenuate MGO-induced cell damage and this effect may be associated with its ability to trap MGO.

Reduced upregulation of the expression of ICAM-1 and TGF- $\beta 1$ with Cur-MGO adducts. The expression levels of ICAM-1 and TGF- $\beta 1$ in HUVECs were measured by immu- nocytochemistry and western blot analysis. As presented in Figs. 8 and 9, MGO (1 mM) or MGO + HSA upregulated the expression levels of ICAM- 1 and TGF- $\beta 1$ in HUVECs following stimulation for $48 \mathrm{~h}$. In addition, incubation with Cur-MGO adducts $\left(10^{-7}, 10^{-6}\right.$ and $\left.10^{-5} \mathrm{M}\right)$ resulted in reduced upregulation in the expression levels of ICAM- 1 and TGF- $\beta 1$ in HUVECs compared with MGO or MGO + HSA $(\mathrm{P}<0.05$ or 
A
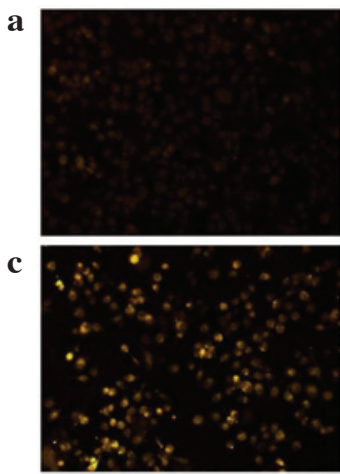

e

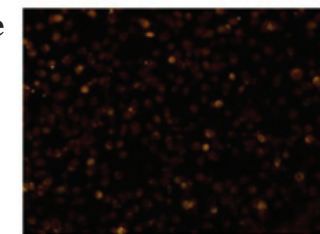

g

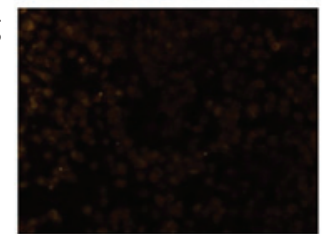

b

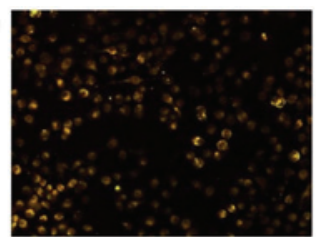

d

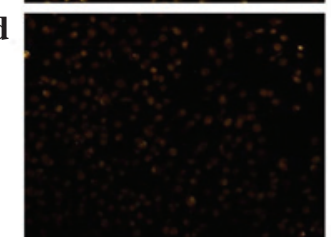

f

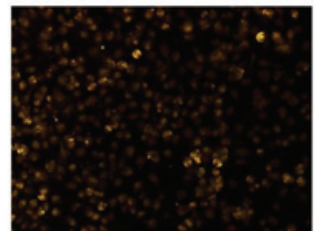

h

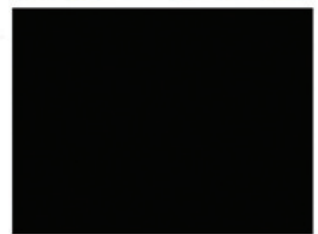

B
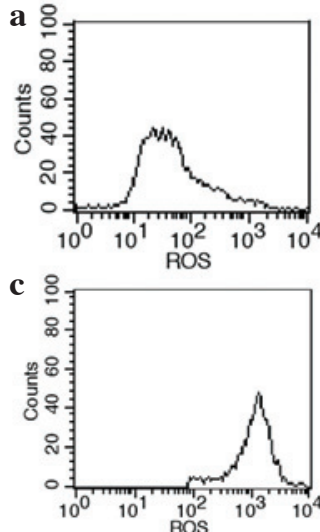

e 을

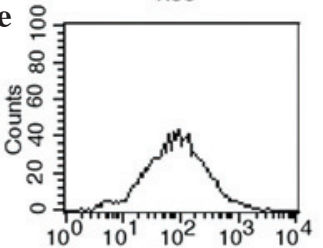

ROS
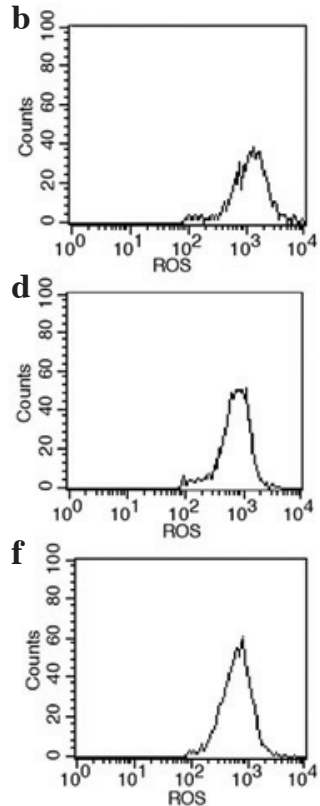
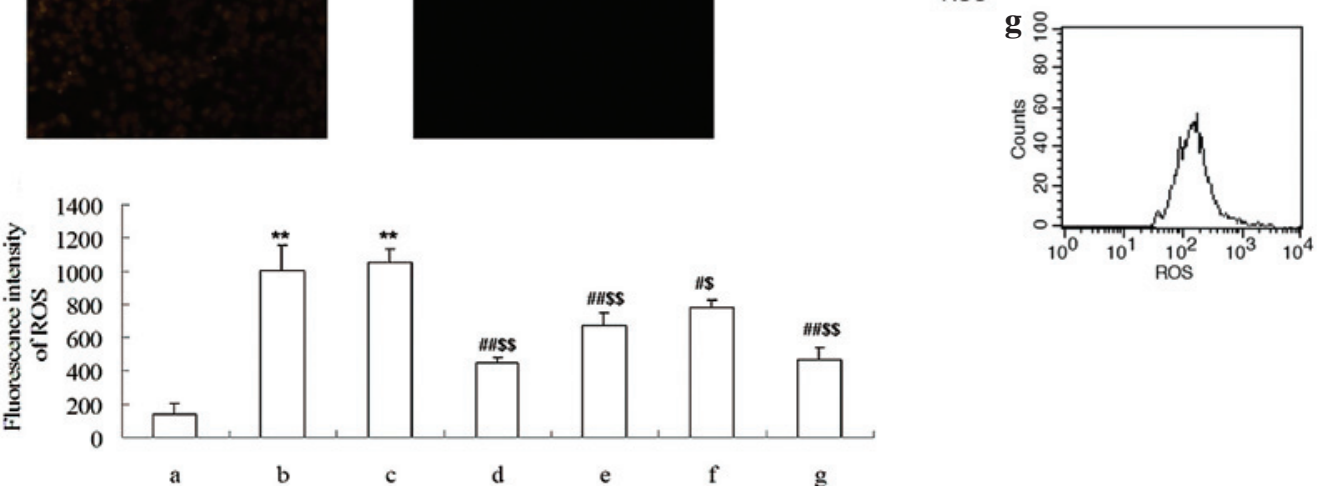

Figure 6. Effect of Cur-MGO adducts on ROS generation in HUVECs. (A) Intracellular ROS was stained with $5 \mu$ M DHE and imaged using fluorescence microscopy. (B) The level of intracellular ROS was measured using flow cytometry and (C) the fluorescence intensity was calculated. a, HSA; b, MGO; c, $\mathrm{MGO}+\mathrm{HSA} ; \mathrm{d}, \mathrm{MGO}+\mathrm{HSA}+\mathrm{Cur}\left(10^{-7} \mathrm{M}\right) ; \mathrm{e}, \mathrm{MGO}+\mathrm{HSA}+\mathrm{Cur}\left(10^{-6} \mathrm{M}\right) ; \mathrm{f}, \mathrm{MGO}+\mathrm{HSA}+\mathrm{Cur}\left(10^{-5} \mathrm{M}\right) ; \mathrm{g}, \mathrm{MGO}+\mathrm{HSA}+\mathrm{AG}\left(10^{-6} \mathrm{M}\right) ; \mathrm{h}$, negative control. The experiments were repeated three times and the data are presented as the mean \pm standard deviation $(\mathrm{n}=3) .{ }^{* * *} \mathrm{P}<0.01$, vs. $\mathrm{HSA}$; ${ }^{\#} \mathrm{P}<0.05$, ${ }^{\# \#} \mathrm{P}<0.01$, vs. MGO; ${ }^{\$} \mathrm{P}<0.01$, vs. HSA + MGO. Cur, curcumin; MGO, methylgloxal; ROS, reactive oxygen species; HUVECs, human umbilical vein endothelial cells; DHE, dihydroethidium; HSA, human serum albumin; AG, aminoguanidine hydrochloride.

$\mathrm{P}<0.01)$. These data indicate that Cur is able to attenuate the effect of MGO on the expression of ICAM- 1 and TGF- $\beta 1$ in HUVECs. The effect of Cur may be associated with its ability to trap the carbonyl compound MGO.

\section{Discussion}

Carbonyl stress-induced oxidative damage and inflammatory responses have been suggested as a potential mechanism contributing to endothelial cell dysfunction (5). Under hyperglycemic conditions, the glucose metabolism may accelerate the abnormal accumulation of the reactive dicarbonyl compound MGO (2). This induces oxidative stress and inflammatory responses, resulting in the initiation and development of diabetic vascular complications (17). Previous studies have demonstrated that natural products are able to attenuate endothelial damage via the capture of carbonyl compounds $(3,7,10)$. Cur, a major active component of Curcuma longa L., has been demonstrated to possess beneficial effects on carbonyl stress-induced endothelial damage (18). Although the trapping

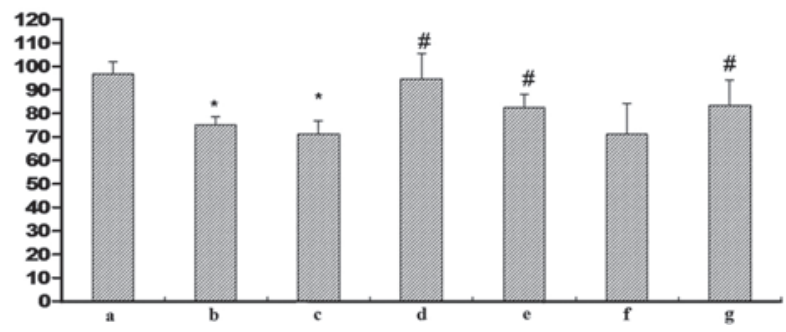

Figure 7. Effect of Cur-MGO adducts on the cell viability of HUVECs. Cells were treated with the reaction solution and the optical density value was determined using an MTT assay. a, HSA; b, MGO; c, MGO + HSA; $\mathrm{d}, \mathrm{MGO}+\mathrm{HSA}+\mathrm{Cur}\left(10^{-7} \mathrm{M}\right) ; \mathrm{e}, \mathrm{MGO}+\mathrm{HSA}+\mathrm{Cur}\left(10^{-6} \mathrm{M}\right) ; \mathrm{f}$, $\mathrm{MGO}+\mathrm{HSA}+\mathrm{Cur}\left(10^{-5} \mathrm{M}\right) ; \mathrm{g}, \mathrm{MGO}+\mathrm{HSA}+\mathrm{AG}\left(10^{-6} \mathrm{M}\right)$. The data are presented as the mean \pm standard deviation $(n=3)$. ${ }^{*} \mathrm{P}<0.01$, vs. HSA; ${ }^{*} \mathrm{P}<0.05$, vs. MGO or MGO + HSA. Cur, curcumin; MGO, methylgloxal; HUVECs, human umbilical vein endothelial cells; HSA, human serum albumin; AG, aminoguanidine hydrochloride.

ability of Cur on MGO is known, the effects of Cur-MGO adducts remain to be fully elucidated. In the present study, due to the instability of the chemical structure of Cur, additional 
A
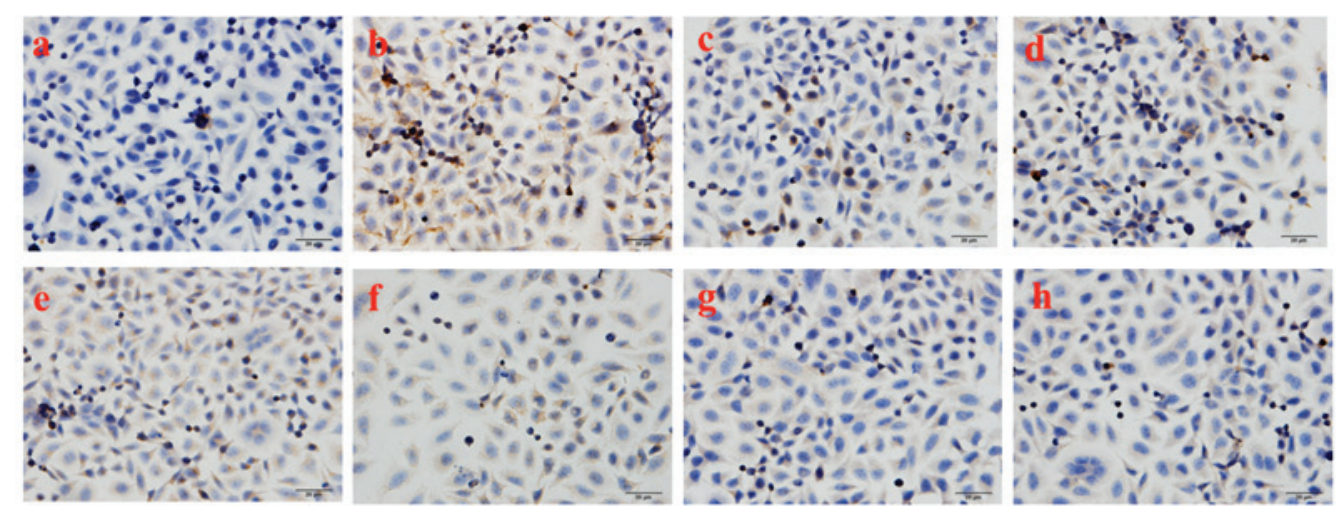

B

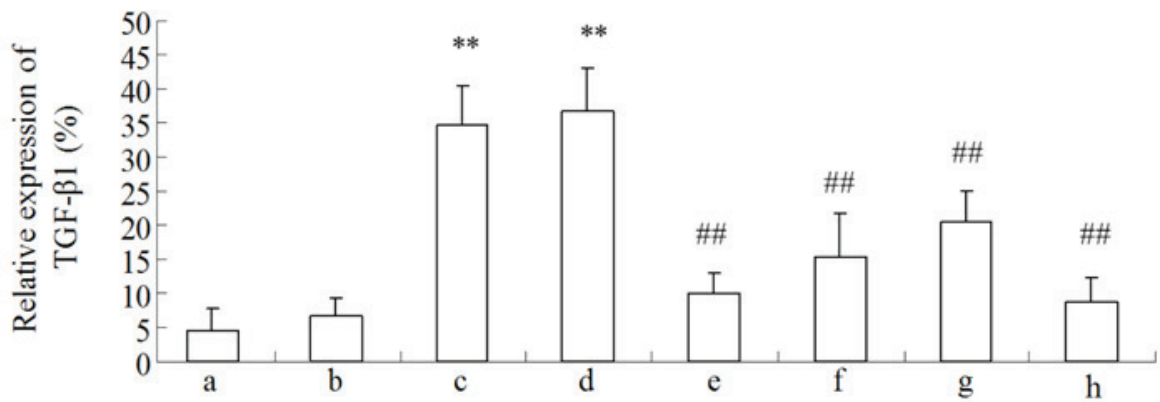

C

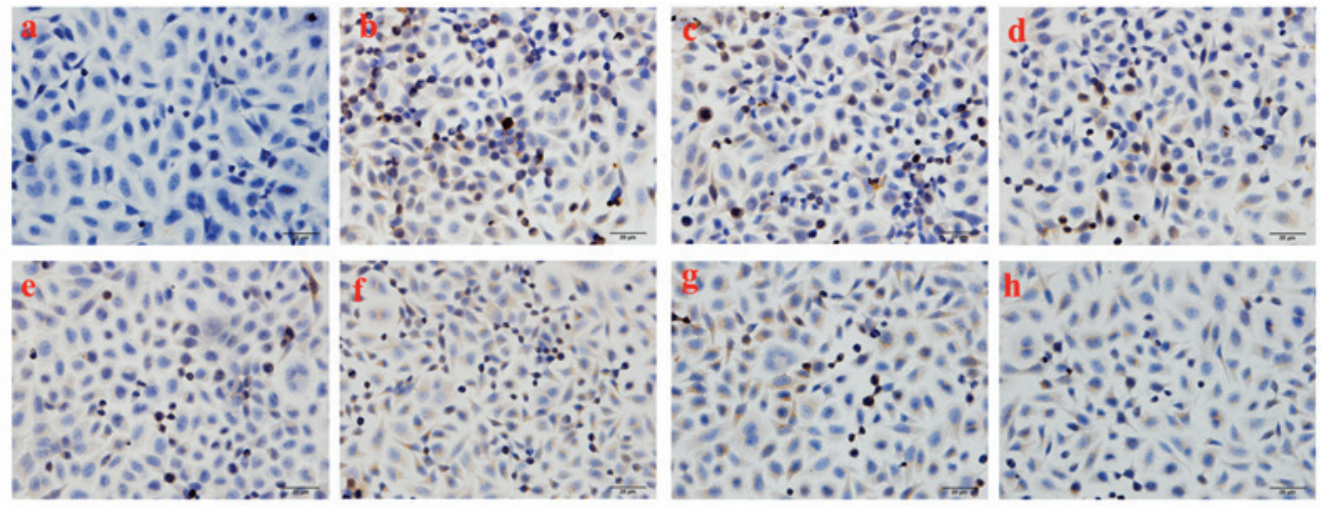

D

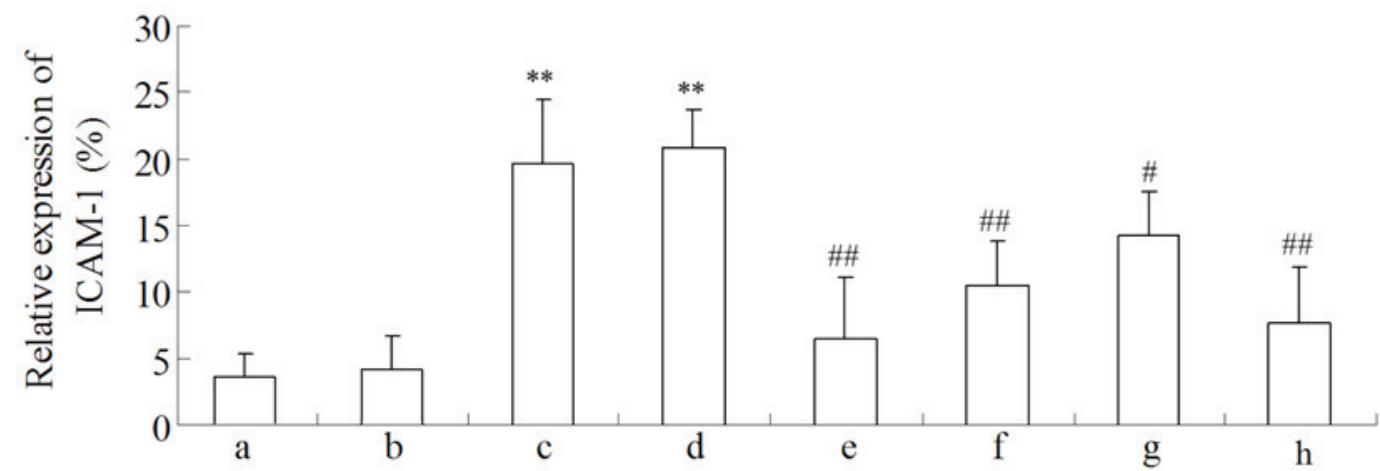

Figure 8. Regulation of Cur-MGO adducts on inflammatory cytokines (A and B) ICAM-1 and (C and D) TGF- $\beta 1$ expression in HUVECs by immunocytochemistry. a, Control; b, HSA; c, MGO; d, MGO + HSA; e-g, MGO + HSA in the presence of $10^{-7}, 10^{-6}$ and $10^{-5} \mathrm{M}$ Cur, respectively; h, MGO + HSA in the presence of $10^{-6} \mathrm{M}$ AG. This experiment was performed three times. The data are presented as the mean \pm standard deviation $(\mathrm{n}=3)$. ${ }^{* *} \mathrm{P}<0.01$, vs. HSA; ${ }^{\#} \mathrm{P}<0.05$ and ${ }^{\# /} \mathrm{P}<0.01$, vs. MGO and HAS+MGO. Cur, curcumin; MGO, methylgloxal; ICAM-1, intercellular adhesion molecule-1; TGF- $\beta 1$, transforming growth factor- $\beta 1$; HUVECs, human umbilical vein endothelial cells; HSA, human serum albumin; AG, aminoguanidine hydrochloride.

Cur-MGO adducts were identified by LC-ESI-MS/MS. In addition, the Cur-MGO adducts were demonstrated to result in a reduction in cell injury compared with MGO alone. These data demonstrate that Cur may attenuate MGO-induced endothelial damage via the trapping of MGO to form a number of Cur-MGO adducts. 
A

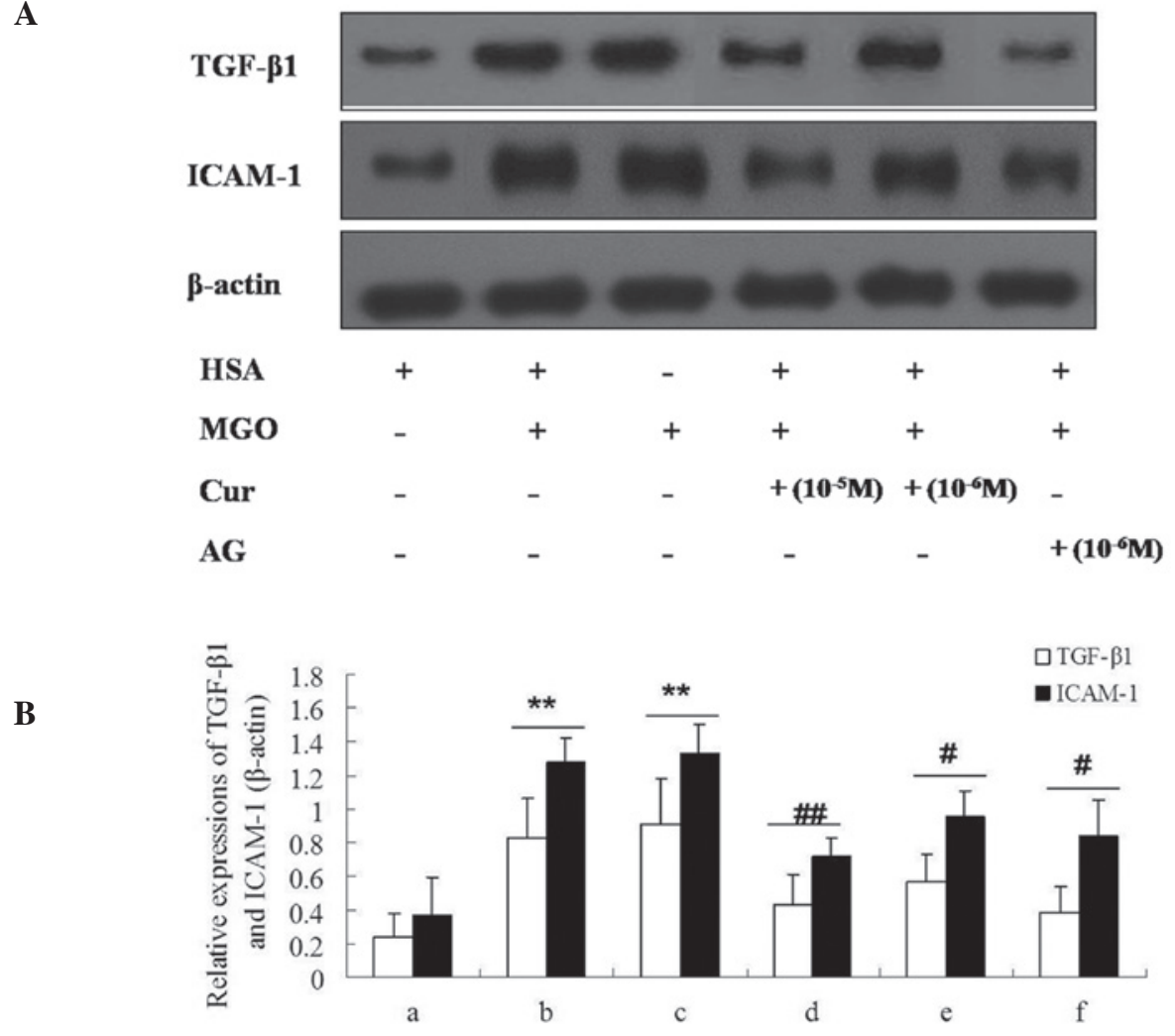

Figure 9. Effect of Cur-MGO adducts on the expression of the inflammatory cytokines ICAM-1 and TGF- $\beta 1$ in HUVECs by western blot analysis. (A) The bands of ICAM-1 and TGF- $\beta 1$; (B) the relative expression levels of ICAM-1 and TGF- $\beta 1$. a, HSA; b, MGO; c, MGO + HSA; d, MGO + HSA + Cur (10-6 M); e, $\mathrm{MGO}+\mathrm{HSA}+\mathrm{Cur}\left(10^{-5} \mathrm{M}\right) ; \mathrm{f}, \mathrm{MGO}+\mathrm{HSA}+\mathrm{AG}\left(10^{-6} \mathrm{M}\right)$. Data are presented as the mean \pm standard deviation $(\mathrm{n}=3) .{ }^{* *} \mathrm{P}<0.01, \mathrm{vs} . \mathrm{HSA} ;{ }^{\#} \mathrm{P}<0.05,{ }^{\# /} \mathrm{P}<0.01$, vs. MGO or MGO + HSA. Cur, curcumin; MGO, methylgloxal; ICAM-1, intercellular adhesion molecule-1; TGF- $\beta 1$, transforming growth factor- $\beta 1$; HUVECs, human umbilical vein endothelial cells; HSA, human serum albumin; AG, aminoguanidine hydrochloride.

Cur is a highly active and unstable compound which is easily oxidized. The rate of degradation of curcuminoids increases with increasing $\mathrm{pH}$ in media (18). In the current study, in order to ensure the reliability of the reaction system, DB was used to investigate the ability of Cur to trap the dicarbonyl compound, MGO. These data demonstrate that the reaction product of DB and MGO matched the 2-methylquinoxaline standard, according to the UV spectra and retention time. These results demonstrate that the reaction system is suitable for trapping the dicarbonyl compound MGO using natural products.

The present study investigated the optimal reaction ratio of MGO and Cur. The reaction of MGO and Cur at the ratios of 1:5, 1:3, 1:1, 3:1 and 5:1 were analyzed using HPLC-DAD, which indicated that a 1:1 ratio of MGO and Cur was best suited for the reaction. The structure of the Cur-MGO adducts which were derived from the condensation of one molecule Cur and one molecule MGO confirmed this was the optimal reaction ratio. Although the reaction time of Cur and MGO was not investigated, the reaction was maintained for $720 \mathrm{~min}$ according to the investigation of the reaction time of $\mathrm{DB}$ and MGO. These conditions were established to be suitable for the reaction of Cur and MGO.

The $-\mathrm{OH}$ and $-\mathrm{OCH}_{3}$ groups on the phenyl ring of Cur possess strong electron-donating abilities and increase the electron density of the benzene ring, resulting in an increase in the electrophilic reactivity of the carbon chain (19). However, the electrophilic reactivity of the carbon chain of Cur is reduced by the loss of the $-\mathrm{OCH}_{3}$ group on the benzene ring with a reduction in electronic capacity. Following the reaction with MGO under experimental conditions, adduct 1 was identified in the reaction system based on the fragment ions. The condensation reaction between Cur and the aldehyde group of MGO resulted in the loss of one molecule of $\mathrm{H}_{2} \mathrm{O}$. The results of the current study are in agreement with previous studies $(6,7,10,18,19)$. In addition, the current study identified two further compounds, adducts 2 and 3. Analysis using HPLC/ESI-MS-MS demonstrated that these two compounds have lost two $-\mathrm{OCH}_{3}$ groups, with 441,411 and $381 \mathrm{~m} / \mathrm{z}$ fragments ions. These data provide evidence that adduct 1 was unstable and easily formed adducts 2 and 3 in the reaction system. However, it remains unclear whether Cur condensates with MGO prior to or following the loss of the $-\mathrm{OCH}_{3}$ groups.

The formation and accumulation of AGEs has been suggested to contribute to endothelial dysfunction. Trapping the dicarbonyl compound MGO has been demonstrated to inhibit the formation of AGEs to attenuate carbonyl stress-induced cell injury (20). In the current study, the inhibition of the formation of AGEs by Cur $\left(10^{-7}, 10^{-6}, 10^{-5} \mathrm{M}\right)$ was investigated from $0-168 \mathrm{~h}$. The kinetic curves of the formation of AGEs indicated that the formation was stable from 24-168 h. Furthermore, the rate of AGE formation was reduced by the Cur-MGO adducts in a concentration-dependent manner, further supported by the ELISA results. The present study provides further evidence for the effect of Cur on the attenuation of endothelial dysfunction via the trapping of MGO to inhibit the formation of AGEs. 
In order to investigate the effect of Cur on the inhibition of the formation of MGO-AGEs by the trapping of MGO, the oxidative damage and inflammatory responses of the reaction product of MGO and HSA in the presence or absence of Cur were compared in the current study. The data collected demonstrate that the cytotoxicity of MGO + HSA in presence of Cur is reduced compared with MGO + HSA alone. The present study provides further evidence for the Cur-mediated attenuation of the pathogenesis and development of endothelial damage via MGO trapping.

Carbonyl stress may induce oxidative stress which is associated with endothelial cell dysfunction. The oxidative modification of HSA by MGO is a causative factor in oxidative injury (21). Increasing evidence demonstrates that MGO stimulated superoxide production from mitochondria and partially stimulated ROS generation in HUVECs $(22,23)$. In the current study, MGO was modified by Cur to form Cur-MGO adducts which were observed to reduce the cytotoxicity of MGO. Notably, there was a significant reduction in the oxidative damage resulting from incubation with Cur-MGO compared with MGO or MGO + HSA. DHE staining and flow cytometry indicated that the trapping of MGO by Cur is a potential mechanism by which carbonyl stress-induced endothelial damage may be ameliorated.

ICAM- 1 and TGF- $\beta 1$ serve important roles in the systemic inflammatory response in generalized endothelial dysfunction (24). In the current study, following exposure of HUVECs to MGO or MGO + HSA, the expression levels of ICAM-1 and TGF- $\beta 1$ were significantly increased, compared with HSA alone. However, following stimulation with Cur-MGO, the expression levels of ICAM- 1 and TGF- $\beta 1$ were reduced compared with MGO + HSA without Cur. These data indicate that the cytotoxicity of MGO was attenuated due to the trapping of MGO by Cur.

Taken together, these data provide evidence demonstrating that Cur attenuates the cytotoxicity of the dicarbonyl compound MGO in endothelial damage through the trapping of MGO. In the current study, the coreaction of Cur with MGO resulted in the formation of Cur-MGO adducts, two of which have not previously been described. The results of LC-ESI-MS/MS indicated that the two novel adducts may be formed due to the instability of adduct 1 or Cur. The differences in oxidative damage and the inflammatory responses to Cur-MGO and MGO + HSA indicate that the attenuation of endothelial damage by Cur may be associated with its ability to trap dicarbonyl compounds, such as MGO. The current study provides further evidence for the protective role of Cur against carbonyl stress-induced endothelial damage.

\section{Acknowledgements}

The current study was supported by grants from the National Natural Science Foundation of China (grant no. 81202906) and the Education Department of Shaanxi Province (grant no. 12JK1019).

\section{References}

1. Sabayan B, Westendorp RG, Grond J, Stott DJ, Sattar N, van Osch MJ, van Buchem MA and de Craen AJ: Markers of endothelial dysfunction and cerebral blood flow in older adults. Neurobiol Aging 35: 373-377, 2014.
2. Žižek B, Žižek D, Bedenčič K, Jerin A and Poredoš P: Effect of metabolic abnormalities on endothelial dysfunction in normotensive offspring of subject with hypertension. Int Angiol 32: 386-393, 2013.

3. Lo CY, Li S, Tan D, Pan MH, Sang S and Ho CT: Trapping reactions of reactive carbonyl species with tea polyphenols in simulated physiological conditions. Mol Nutr Food Res 50: 1118-1128, 2006

4. van Eupen MG, Schram MT, Colhoun HM, Hanssen NM, Niessen HW, Tarnow L, Parving HH, Rossing P, Stehouwer CD and Schalkwijk CG: The methylglyoxal-derived AGE tetrahydropyrimidine is increased in plasma of individuals with type 1 diabetes mellitus and in atherosclerotic lesions and is associated with sVCAM-1. Diabetologia 56: 1845-1855, 2013.

5. Akhand AA, Hossain K, Mitsui H, Kato M, Miyata T, Inagi R, Du J, Takeda K, Kawamoto Y, Suzuki H, et al: Glyoxal and methylglyoxal trigger distinct signals for map family kinases and caspase activation in human endothelial cells. Free Radic Biol Med 31: 20-30, 2001.

6. Santel T, Pflug G, Hemdan NY, Schäfer A, Hollenbach M, Buchold M, Hintersdorf A, Lindner I, Otto A, Bigl M, et al: Curcumin inhibits glyoxalase 1: A possible link to its anti-inflammatory and anti-tumor activity. PLoS One 3: e3508, 2008.

7. Liu JP, Feng L, Zhu MM, Wang RS, Zhang MH, Hu SY, Jia XB and $\mathrm{Wu} \mathrm{JJ}$ : The in vitro protective effects of curcumin and demethoxycurcumin in Curcuma longa extract on advanced glycation end products-induced mesangial cell apoptosis and oxidative stress. Planta Med 78: 1757-1760, 2012.

8. Hu TY, Liu CL, Chen JY and Hu ML: Curcumin ameliorates methylglyoxal-induced alterations of cellular morphology and hyperpermeability in human umbilical vein endothelial cells. J Funct Foods 5: 745-754, 2013.

9. Lip H, Yang K, MacAllister SL and O'Brien PJ: Glyoxal and methylglyoxal: Autoxidation from dihydroxyacetone and polyphenol cytoprotective antioxidant mechanisms. Chem Biol Interact 202: 267-274, 2013

10. Hu TY, Liu CL, Chyau CC and Hu ML: Trapping of methylglyoxal by curcumin in cell-free systems and in human umbilical vein endothelial cells. J Agric Food Chem 60: 8190-8196, 2012.

11. Lv L, Shao X, Chen H, Ho CT and Sang S: Genistein inhibits advanced glycation end product formation by trapping methylglyoxal. Chem Res Toxicol 24: 579-586, 2011.

12. Schmitt A, Bigl K, Meiners I and Schmitt J: Induction of reactive oxygen species and cell survival in the presence of advanced glycation end products and similar structures. Biochim Biophys Acta 1763: 927-936, 2006.

13. Feng L, Zhu M, Zhang M, Gu J, Jia X, Tan X, Gao C and Zhu Q: The protection of 4,4'-diphenylmethane-bis(methyl) carbamate from Cortex Mori on advanced glycation end product-induced endothelial dysfunction: Via inhibiting AGE formation or blocking AGEs-RAGE axis? Fitoterapia 89: 239-249, 2013.

14. Feng L, Zhu MM, Zhang MH, Wang RS, Tan XB, Song J, Ding SM, Jia XB and Hu SY: Protection of glycyrrhizic acid against AGEs-induced endothelial dysfunction through inhibiting RAGE/NF- $\kappa$ B pathway activation in human umbilical vein endothelial cells. J Ethnopharmacol 148: 27-36, 2013.

15. Li Y, Gu JF, Zou X, Wu J, Zhang MH, Jiang J, Qin D, Zhou JY, Liu BX, Zhu YT, et al: The anti-lung cancer activities of steroidal saponins of P. polyphylla Smith var. chinensis (Franch.) Hara through enhanced immunostimulation in experimental Lewis tumor-bearing C57BL/6 mice and induction of apoptosis in the A549 cell line. Molecules 18: 12916-12936, 2013.

16. Feng L, Zhu M, Zhang M, Jia X, Cheng X, Ding S and Zhu Q: Amelioration of compound 4,4'-diphenylmethane-bis(methyl) carbamate on high mobility group box1-mediated inflammation and oxidant stress responses in human umbilical vein endothelial cells via RAGE/ERK1/2/NF- $\kappa$ B pathway. Int Immunopharmacol 15: 206-216, 2013.

17. Li W, Maloney RE, Circu ML, Alexander JS and Aw TY: Acute carbonyl stress induces occludin glycation and brain microvascular endothelial barrier dysfunction: Role for glutathione-dependent metabolism of methylglyoxal. Free Radic Biol Med 54: 51-61, 2013.

18. Singh R, Kristensen S and Tønnesen HH: Influence of vehicle properties and excipients on hydrolytic and photochemical stability of curcumin in preparations containing Pluronics: Studies of curcumin and curcuminoids XLVIII. Pharmazie 68: 160-169, 2013.

19. Deshpande SS and Maru GB: Effects of curcumin on the formation of benzo[a]pyrene derived DNA adducts in vitro. Cancer Lett 96: 71-80, 1995. 
20. Okouchi M, Okayama N and Aw TY: Preservation of cellular glutathione status and mitochondrial membrane potential by $\mathrm{N}$-acetylcysteine and insulin sensitizers prevent carbonyl stress-induced human brain endothelial cell apoptosis. Curr Neurovasc Res 6: 267-278, 2009.

21. Ciolino HP and Levine RL: Modification of proteins in endothelial cell death during oxidative stress. Free Radic Biol Med 22: 1277-1282, 1997.

22. Tatsunami R, Oba T, Takahashi K and Tampo Y: Methylglyoxal causes dysfunction of thioredoxin and thioredoxin reductase in endothelial cells. J Pharmacol Sci 111: 426-432, 2009.
23. Miyazawa N, Abe M, Souma T, Tanemoto M, Abe T, Nakayama M and Ito S: Methylglyoxal augments intracellular oxidative stress in human aortic endothelial cells. Free Radic Res 44: 101-107, 2010.

24. Szarka A, Rigó J Jr, Lázár L, Beko G and Molvarec A: Circulating cytokines, chemokines and adhesion molecules in normal pregnancy and preeclampsia determined by multiplex suspension array. BMC Immunol 11: 59, 2010 